\title{
Long-term expression of metabolism-associated genes in the rat hippocampus following recurrent neonatal seizures and its regulation by melatonin
}

\author{
HONG NI $^{1}$, QI SUN ${ }^{1}$, TIAN TIAN ${ }^{1}$, XING FENG $^{1}$ and BAO-LIANG SUN ${ }^{2}$ \\ ${ }^{1}$ Neurology Laboratory, Institute of Pediatrics, Children's Hospital of Soochow University, Suzhou, Jiangsu 215003; \\ ${ }^{2}$ Department of Neurology, Key Laboratory of Cerebral Microcirculation, University of Shandong, \\ Affiliated Hospital of Taishan Medical College, Taian, Shandong 271000, P.R. China
}

Received July 16, 2014; Accepted March 23, 2015

DOI: $10.3892 / \mathrm{mmr} .2015 .3691$

\begin{abstract}
Despite the effective use of antiepileptic drugs (AEDs) for epilepsy, therapeutic failure occurs in $30 \%$ of patients. Novel approaches are targeting the inhibition of epileptogenesis. N-acetyl-5-methoxytryptamine (melatonin) is an indoleamine produced mainly by the pineal gland, and has been observed to exhibit antiepileptic and neuroprotective effects in experimental and clinical investigations. In the present study, the underlying protective mechanism of melatonin on neonatal seizure-induced long-term excitotoxicity was examined in the hippocampus of rats, predominantly on the metabolism-associated genes. Sprague Dawley rats (6-day-old; P6) were randomly divided into four groups, the control (Cont), melatonin-treated control (Mel), recurrent neonatal seizure (RS) and treatment with melatonin and RS combined (Mel+RS). At P35, mossy fiber sprouting and changes in gene expression in hippocampus were assessed using Timm staining, reverse transcription-quantitative polymerase chain reaction and use of the $2^{-\Delta C T}$ methods, respectively. The aberrant mossy fiber sprouting in the supra granular region of the dentate gyrus and CA3 subfield of the hippocampus was suppressed by pretreatment with melatonin. In addition, among the nineteen genes identified, four energy metabolism-associated genes (Kcnj11, leptin receptor, dopamine receptor D2 and melanocortin 4 receptor), four lipid metabolism-associated genes (apolipoprotein A-I, opioid receptor $x 1$, pyruvate dehydrogenase kinase, isozyme 4 and cytochrome P450, family 46, subfamily a, polypeptide 1) and zinc transporter 1 (ZnT1), sphingomyelinase (nSMase) and Cathepsin-E, were markedly downregulated by melatonin
\end{abstract}

Correspondence to: Dr Hong Ni, Neurology Laboratory, Institute of Pediatrics, Children's Hospital of Soochow University, 303 Jingde Road, Suzhou, Jiangsu 215003, P.R. China

E-mail:nhdoctor@126.com

Key words: gene expression, hippocampus, mossy fiber spouting, melatonin, neonatal seizure treatment in the Mel group or in the developmental seizure RS and Mel+RS groups, compared with that in the Cont group. Furthermore, the melatonin-pretreated seizure rats (Mel+RS) exhibited a significantly upregulated expression of calcium/calmodulin-dependent protein kinase II $\alpha$ (CaMKII $\alpha$ ), acetyl-Coenzyme A acetyltransferase 1 (ACAT1), ZnT-1, metallothionein 1 (MT-1), nSMase and Cathepsin-E, compared with the RS rats. Thus, the present study investigated changes in the expression of metabolic genes in the hippocampus following pretreatment with melatonin. Fluorthyl-induced decreases in the expression levels of ACAT1/nSMase/Cathepsin-E, ZnT-1/MT-1 and CaMKII $\alpha$ in the hippocampus, and the reversal by melatonin may be associated with a decrease in neonatal seizure-induced aberrant mossy fiber sprouting, which requires further investigation.

\section{Introduction}

Epilepsy is a relatively common neurologic disorder in children (1). Although conventional anti-epileptic drugs (AEDs) control the disorder in the majority of patients, they fail to provide therapeutic benefit in $20-25 \%$ of patients (2). In addition, the serious adverse effects of AEDs on brain development, particularly on cognitive function, has provoked further research on non-AED management modalities. A number of previous studies have investigated alternative treatments for epilepsy, including the use of melatonin, a ketogenic diet, vitamins or biofeedback $(3,4)$. Among these, melatonin has been used as an adjunct treatment for pediatric epilepsy.

Melatonin is a hormone, which is made in the pineal gland. Apart from its well-recognized roles as an anti-aging substance, sleep aid and antioxidant, clinical data suggests that it is effective in the adjunctive therapy of osteoporosis, sepsis, jet-lag syndrome, neurodegenerative diseases, certain types of isomnia and even cancer (3). As for epilepsy, melatonin may have clinical benefits. In a previous study, 10 paediatric patients suffering from severe epileptic disorders were administered a nightly dose of $3 \mathrm{mg}$ melatonin. The results demonstrated regained periodic plasma melatonin levels and improved control in convulsive episodes (5). Baseline melatonin levels may be low in childhood refractory epilepsy 
and febrile seizures, and levels increase markedly following seizures (6-8). Another study demonstrated that treatment with melatonin (10 mg daily at night) decreased diurnal seizures in 10 patients aged between 9 and 32 years with intractable epilepsy (9). The majority of experimental data also indicates the anticonvulsant properties of the hormone in maximal electroshock, amygdale kindling, pentylenetetrazole (PTZ), pilocarpine, penicillin or kainic- and quinolinic acid-induced animal models of epilepsy (10). However, the suggestion that melatonin has anticonvulsant properties remains controversial. For example, oral melatonin (5 mg) in neurologically disabled children was observed to result in increased seizures 13 days following the onset of the therapy. The proconvulsant effects disappeared immediately following treatment discontinuation (11). In a hippocampal slice seizure model, induced using low $\mathrm{Mg}^{2+}$ or bicuculline, the pharmacological concentration of melatonin enhanced the frequency of epileptiform activity, whereas this effect was suppressed by luzindole, a melatonin antagonist (12). These results suggest that further investigation is required prior to establishing melatonin as a potential drug candidate for adjunctive therapy in children with epilepsy.

In the majority of experimental seizure models, the anticonvulsant action of melatonin has been observed in adult animals in rats, mice, hamster and guinea pigs. However, there has been little investigation of the effects of melatonin in developmental animals. Using a phenobarbital-induced neonatal seizure rat model, Forcelli et al observed that melatonin $(0-80 \mathrm{mg} / \mathrm{kg})$, prior to PTZ, potentiated the anticonvulsant efficacy of phenobarbital, however, it did not exert anticonvulsant effects alone. This previous study did not further investigate the underlying molecular mechanisms (13). Our previous study investigated the dynamic expression pattern of mossy fiber sprouting-associated genes in the rat brain using a flurothyl-induced recurrent neonatal seizure model. Furthermore, this model, as described extensively, has been widely used to evaluate the neuroprotective efficacy of compounds, including autophagy inhibitor 3-MA, CBI and E-64d (14-16). The important function of melatonin in epilepsy, and the requirement to elucidate its role in neonatal seizure-induced long-term excitotoxicity, prompted the present study to further examine whether pretreatment with melatonin alleviates the deleterious changes in the hippocampus. These changes are indicated by hippocampal mossy fiber sprouting and metabolic-associated genes, which are integral components of developmental brain injury-induced epileptogenesis $(14,15)$.

\section{Materials and methods}

Animal preparation. Sprague-Dawley rats were treated in accordance with the guidelines set by the National Institutes of Health for the humane treatment of animals. The litters were randomly assigned to an experimental group and the animals were weaned on postnatal day 21 , and following this age they were housed in a standard light-dark cycle. The present study was approved by the ethics committee of Soochow University Affiliated Children's Hospital (Suzhou, China). Attempts were made to minimize the number of animals used. A total of 48 Sprague-Dawley neonatal rats at postnatal day 6 (P6) were randomly divided into four groups: Control (Cont), melatonin-treated control $(\mathrm{Mel})$, recurrent neonatal seizure
(RS) and melatonin and RS combined treatment (Mel+RS). The procedure of seizure induction was described in detail previously (16). In brief, the neonatal rats were placed into a transparent plastic airtight box and liquid volatile flurothyl (bis-2,2,2-triflurothyl ether; Sigma-Aldrich, St. Louis, MO, USA) was added, using a syringe, onto filter paper in the center of the container, for agent evaporation. The rats were immediately removed from the chamber on observation of tonic extension of the forelimbs and hindlimbs. The experimental rats received 45 induced seizures during over nine consequetive days, between P6 and P14. The rats had five seizures/day over this duration, with 30 min intervals between each seizure. The control rats were placed into the container for an equal duration without exposure to the flurothyl. In the two melatonin-treated groups, each rat was pretreated with an intraperitoneal (i.p) injection of melatonin prior to seizure induction $(55 \mathrm{mg} / \mathrm{kg}$, Sigma-Aldrich, St. Louis, MO, USA) $(17,18)$.

For molecular investigations, the animals were sacrificed by decapitation on P35 for subsequent Timm staining and reverse transcription-quantitative polymerase chain reaction (RT-qPCR) analyses.

Timm staining. A total of six rats from each group were anesthetized using chloropent ( $3 \mathrm{ml} / \mathrm{kg}$; i.p; Sinopharm Chemical Reagent Shanghai Co., Ltd., Shanghai, China). The method used has been described in detail previously (19). Rats from each group were perfused through the heart with $0.9 \%$ saline, followed by $0.375 \%$ sodium sulfide and $4 \%$ paraformaldehyde in PBS. The brains were sectioned into $30 \mu \mathrm{m}$-thick coronal tissue sections from the septal area, where the two blades of the hippocampal dentate and pyramidal CA3 region were equal. The processing solutions consisted of $30 \%$ gum Arabic, $3.825 \%$ citric acid, $3.525 \%$ sodium citrate, $3.525 \%$ hydroquinone and $25.5 \%$ silver nitrate. Timm staining was analyzed under a x10 objective on a light microscope.

$R T$ - $q P C R$. A total of six rats from each group were anesthetized using chloropent $(3 \mathrm{ml} / \mathrm{kg}$; i.p.). The method has been described in detail previously (19). The total RNA was extracted from each fresh hippocampal sample using TRIzol reagent (Invitrogen Life Technologies, Carlsbad, CA, USA). The concentration, purity and quantitiy of the total isolated RNA was determined by ultraviolet spectrophotometry, which were of high quality and purity by measuring the optical density at 260 and $280 \mathrm{~nm}$. The total RNA $(2 \mu \mathrm{g})$ was reverse transcribed into cDNA using random primers, 200 units of MMLV reverse transcriptase (Invitrogen Life Technologies), $0.5 \mathrm{mM}$ dNTPs, $10 \mathrm{mM}$ dithiothreitol and 25 unitsof RNase inhibitor (Invitrogen Life Technologies). RT reaction (40 $\mu \mathrm{l})$ was performed at $37^{\circ} \mathrm{C}$ for $60 \mathrm{~min}$, then at $95^{\circ} \mathrm{C}$ for $5 \mathrm{~min}$. RT-qPCR was performed using TaqMan probe-based chemistry (Applied Biosystems, Foster City, CA, USA). The primers and probes of the 19 genes were designed against GenBank-published sequences using Primer Express 2.0 software (Applied Biosystems Life Technologies, Inc., Foster City, CA, USA). The sequences are listed in the subsequent figures. The 19 genes were as follows: Kcnj11, Lepr, Drd2, $\mathrm{Mc} 4 \mathrm{r}$ and $\mathrm{CCK}$, which are involved in regulation of energy metabolism; Apoa1, Oprk1, Pdk4, Cyp46a1, ACAT1, nSMase and Cathepsin-E, which are involved in regulation of lipid 


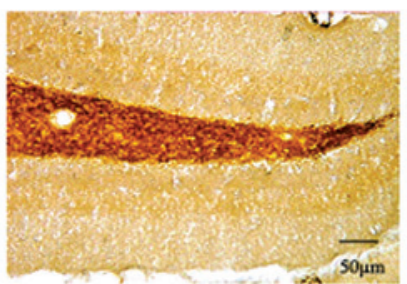

A1

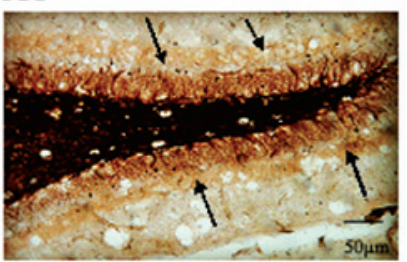

C1

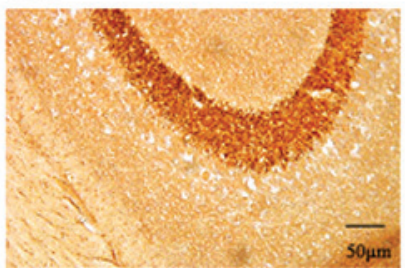

A2

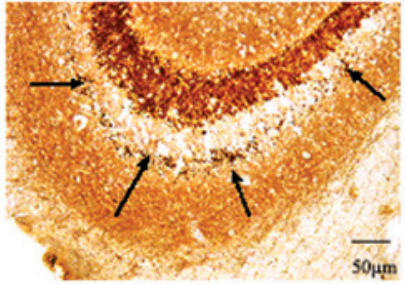

$\mathrm{C} 2$

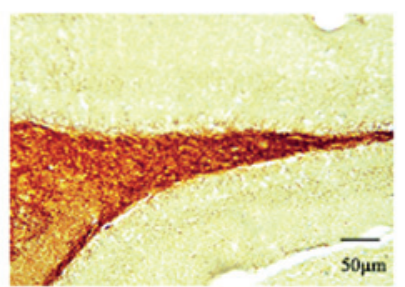

B1

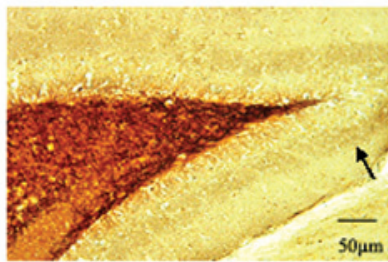

D1

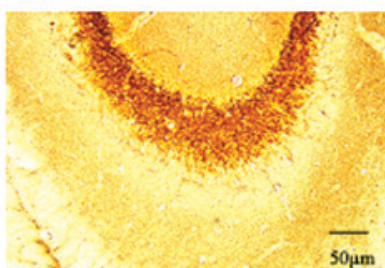

B2

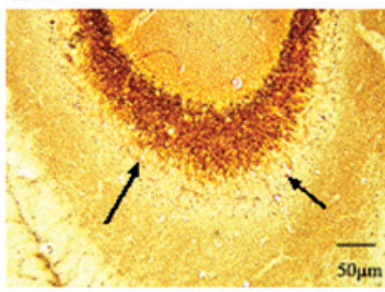

D2

Figure 1. Example of mossy fiber sprouting using Timm staining in the (A1 and A2) Cont, (B1 and B2) Mel, (C1 and C2) RS and (D1 and D2) Mel+RS groups. A1-D1 represent the dentate gyrus subfield. A2-D2 represent the CA3 subfield from the Cont and Mel+RS groups, respectively. Excessive Timm staining was observed in the inner molecular layer of the $(\mathrm{C} 1)$ granule cells and the (C2) stratum pyramidale of the CA3 subfield in the RS group (arrows). Scale bar $=50 \mu \mathrm{m}$. Cont, untreated control; Mel, melatonin-treated; RS, recurrent neonatal seizure.

metabolism; NR1, NR2B, GABA-A- $\alpha 1$, CaMKII alpha and beta, which are involved in the regulation of neural excitability; ZnT-1 and MT-1, which are involved in the regulation of zinc metabolism. The RT-qPCR threshold cycle (CT) of the target mRNAs and the internal control $\beta$-actin was determined and the $\Delta \mathrm{CT}$ method of relative quantification was used to determine the fold changes in expression. The ratios of the target gene to $\beta$-actin were calculated as follows: $2^{\mathrm{CT}(\text { target) }-\mathrm{CT}(\beta \text {-actin) }}$. $\Delta \mathrm{CT}=\mathrm{CT}$ target $-\mathrm{CT} \beta$-actin). The fold change in expression was then obtained using the $2^{-\triangle \mathrm{CT}}$ method (20).

The gene expression levels $\left(2^{-\Delta C T}\right)$ were compared with post-hoc comparisons using a Bonferroni test following analysis of variance using SAS 8.0 statistical software. Data are presented as the mean \pm standard deviation. $\mathrm{P}<0.05$ was considered to indicate a statistically significant difference.

\section{Results}

Timm staining. The results of the Timm staining revealed a prominent aggregation of mossy fiber terminals in the

supragranular region of the dentate gyrus (Fig. 1C1) and CA3 subfield (Fig. 1C2). The aberrant mossy fiber sprouting in the dentate gyrus (Fig. 1D1) and in the stratum pyramidale of the CA3 subfield of the hippocampus (Fig. 1D2) was significantly inhibited following pretreatment with melatonin. No mossy fiber terminals were observed in the two control hippocampi (Fig. 1A and B).

RT-PqCR analysis. From the 19 genes examined, four energy metabolism-associated genes (Kcnj11, Lepr, Drd2 and Mc4r; Fig. 2), four lipid metabolism-associated genes (Apoa1, Oprk1, Pdk4 and Cyp46a1; Fig. 3), and ZnT-1, nSMase and Cathepsin-E (Fig. 4), were markedly downregulated in the Mel group and in the RS and Mel+RS groups, compared with those in the Cont group. However, a significant upregulation was observed in the mRNA expression levels of CaMKII $\alpha$, ACAT1, ZnT-1, MT-1, nSMase and Cathepsin-E in the hippocampus of the rats in the Mel+RS group, compared with those in the RS group (Figs. 4 and 5).

\section{Discussion}

The results of the present study indicated that the animals which underwent pretreatment with melatonin exhibited a minor grade of mossy fiber sprouting in the hippocampus, compared with those in the RS group. In addition to this morphological change, the gene expression analysis of a range of hippocampal metabolism-associated genes demonstrated that pretreatment with melatonin led to a significant upregulation of the mRNA expression levels of CaMKII $\alpha$, ACAT1, ZnT-1, MT-1, nSMase and Cathepsin-E in the hippocampi of rats in the Mel+RS group, compared with the RS group.

Hippocampal signaling pathways have been extensively investigated and are considered the predominant target of melatonin for preventing and treating neurological diseases and injuries, including autism, cerebral ischemia and cognitive impairment (21-25). In terms of epilepsy, several independent studies have investigated the effects of melatonin on biochemical alterations in experimental epilepsy models. In a kainate (KA) model of temporal lobe epilepsy, Tchekalarova et al demonstrated that melatonin reduces neuronal damage in the CA1 area of the hippocampus and piriform cortex, and recovers the decrease in hippocampal levels of serotonin (5-HT) in rats with epilepsy (26). Jain et al provided evidence that KA and melatonin-treated animal groups exhibit reduced numbers of nicotinamide adenine dinucleotide phosphate reduced diaphorase positive neurons in the dentate gyrus, hilus, CA1 and $\mathrm{CA} 3$ areas of the hippocampus and a decline in cytosolic $\mathrm{Ca}^{2+}$ concentrations compared with treatment with KA alone, suggesting enhanced levels of cytosolic $\mathrm{Ca}^{2+}$ and nitric oxide (NO) in KA-induced excitotoxicity (27). Mareš et al demonstrated that flurothyl-induced single tonic-clonic seizures caused significant increases in hydroxyl and nitroxyl radicals 60 min following the seizure, which was inhibited following pretreatment with melatonin prior to seizure induction, compared with animals without pretreatment (28). In addition, Atanasova et al observed that pretreatment with melatonin $(10 \mathrm{mg} / \mathrm{kg}$ per day for 14 days) attenuates the KA-induced increase in the level of lipid peroxidation, superoxide dismutase/CuZn production and expression of heat shock 


0.009
0.008

Figure 2. RT-qPCR analysis of hippocampal energy metabolism-associated genes (Kcnj11, Lepr, Drd2, Mc4r and CCK). On comparing the mRNA levels between the Cont group and the three treatment groups, Kcnj11, Lepr, Drd2 and Mc4r were significantly different ("P $<0.05$, compared with the Cont group). Data are presented as the mean \pm standard deviation. The lower part of the chart shows the oligonucleotide primers used for the RT-qPCR analysis. RT-qPCR, reverse transcription-quantitative polymerase chain reaction; Cont, untreated control; Mel, melatonin treatment; RS, recurrent neonatal seizure; Kcnj11, adenosine triphosphate-sensitive potassium channel subunit; Lepr, leptin receptor; Drd2, dopamine receptor D2; Mc4r, melanocortin 4 receptor; CCK, cholecystokinin; CT, threshold cycle.

protein 72 in the hippocampus (29). Taken together, the above observations suggest that the efficacy of melatonin exposure prior to seizures may be associated with the expression of genes in the hippocampus, particularly oxidative stress-associated genes. However, the key signaling molecules underlying its efficacy remain to be elucidated. Due to the anticonvulsant properties of melatonin and that hippocampal mossy fiber sprouting is an integral component of brain injury-induced epileptogenesis, the biochemical processes of melatonin may be elucidated by analyzing the expression of sprouting-associated genes in the hippocampus following seizures.

In our previous study, the abnormal expression of zinc/lipid transporter-associated genes in hippocampus was observed, which may be associated with mossy fiber sprouting following penicillin-induced developmental seizures. The results revealed upregulated expression levels of ACAT1, clusterin and ApoE, and the downregulated expression of ZnT-1 following developmental seizures, compared with the control animals. In addition, the upregulation of ApoE and Clusterin was inhibited following pretreatment with E-64d antophagy inhibitor (15). However, whether melatonin exerts its anticonvulsant effects by regulating lipid/zinc metabolism-associated pathway in the hippocampus has not been previously investigated.

The results of the present study demonstrated, by analyzing the expression pattern of a number of energy/lipid metabolism-associated genes, that the transcriptional regulation of several metabolism-associated molecules, including ACAT1/nSMase/Cathepsin-E and ZnT-1/MT-1, and with CaMKII $\alpha$, may be important for the neuroprotective effect of melatonin following neonatal seizures. Using a flurothy-induced prolonged neonatal seizure model, in which the rats inhaled flurothyl continuously for $30 \mathrm{~min} /$ day for six consecutive days, and semi-quantitative PCR, our previous study revealed the downregulated expression levels of ZnT-1 and CaMKII $\alpha$ in the hippocampus, which may be associated with long-term cognitive deficits and hippocampal mossy 


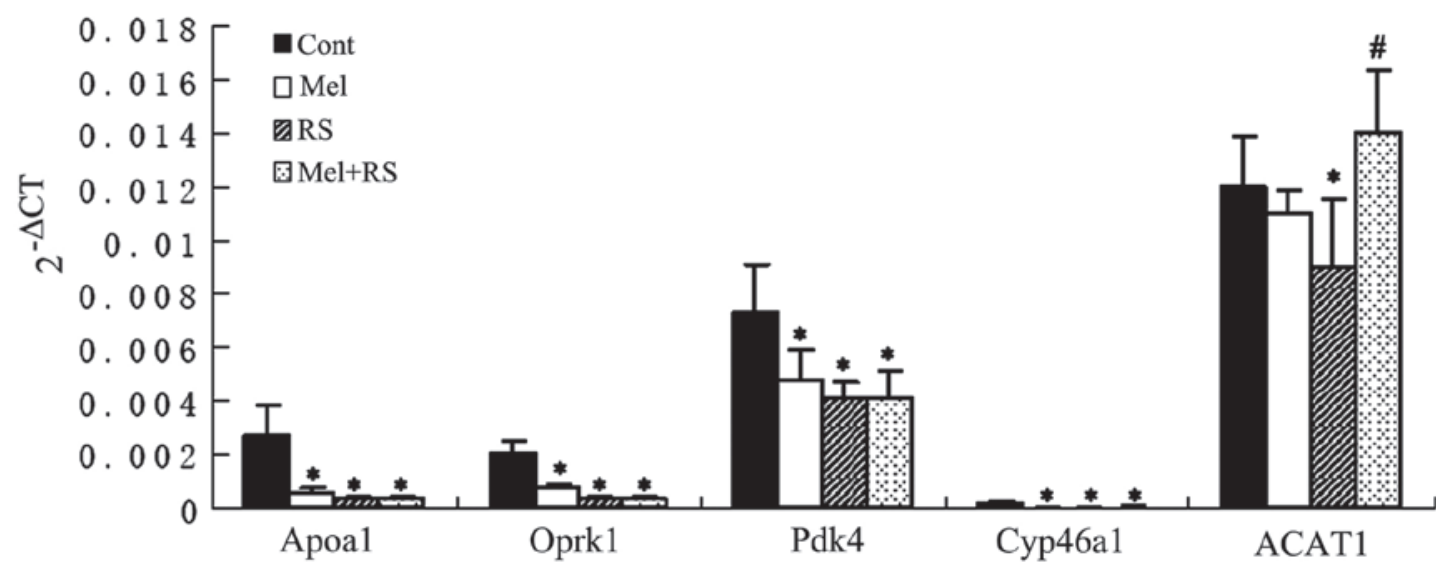

\begin{tabular}{|c|c|c|}
\hline Gene & $\begin{array}{c}\text { GenBank accession } \\
\text { number }\end{array}$ & Primer sequence \\
\hline \multirow[t]{3}{*}{ Apoa1 } & NM_012738 & F:5'-CGATCAGATGCGCGAGAAC-3' \\
\hline & & R:5'-TACTCGATCAGGGTAGGGTGGTT-3' \\
\hline & & Probe:5'-FAM-CCCAGCGCCTGACCGAGATCAA-TAMRA-3' \\
\hline \multirow[t]{3}{*}{ Oprk1 } & NM_017167 & F:5'-TGATGAGTGTGGACCGCTACA-3' \\
\hline & & R:5'-CAAAGGTGTTCGGAAATCCAA-3' \\
\hline & & Probe:5'-FAM-TGCCGTGTGCCACCCTGTGAA-TAMRA-3' \\
\hline \multirow[t]{3}{*}{ PDK4 } & NM_053551 & F:5'-GCTCACACAAGTCAATGGAAAATT-3' \\
\hline & & R:5'-ATGTGGTGAAGGTGTGAAGGAA-3' \\
\hline & & Probe:5'-FAM-CCAGGCCAACCAATCCACATCGTG-TAMRA-3' \\
\hline \multirow[t]{3}{*}{ Сур46а1 } & NM_001108723 & F: 5'-AGTAGTGATTGATGAGGAA-3' \\
\hline & & R: 5'-CTGGAGACAATAACTGATG-3' \\
\hline & & P: 5'-FAM-CACTGCTTACCTGCTCACTCC-TAMRA-3' \\
\hline \multirow[t]{3}{*}{ ACAT1 } & NM_017075 & F: 5'- AAAGAAAACGGCACAGTA -3' \\
\hline & & R: 5’- CAGTGGCTTAACCTTGAG -3' \\
\hline & & probe:5'-FAM-CACACTGAACGACGGAGCA-TAMRA -3' \\
\hline
\end{tabular}

Figure 3. RT-qPCR analysis of hippocampal lipid metabolism-associated genes (Apoa1, Oprk1, Pdk4, Cyp46a1 and ACAT1). On comparing the mRNA levels between the Cont group and the other three groups, significant differences were observed in the expression levels of Apoa1, Oprk1, Pdk4 and Cyp46a1 ( $\mathrm{P}<0.05$, compared with the Cont group). Notably, the expression of ACAT1 in the RS group was significantly downregulated, compared with the Cont group, however, the mRNA level was increased in the Mel+RS group, compared with the RS group ( $\left.{ }^{\#}<0.05\right)$. Data are presented as the mean \pm standard deviation. The lower part of the chart shows the oligonucleotide primers used for real-time RT-qPCR analysis. RT-qPCR, reverse transcription-quantitative polymerase chain reaction; Cont, untreated control; Mel, melatonin treatment; RS, recurrent neonatal seizure. Apoa1, apolipoprotein A-I; Oprk1, opioid receptor $\kappa$ 1; Pdk4, dehydrogenase kinase, isozyme 4; Cyp46a1, cytochrome P450, family 46, subfamily a, polypeptide 1; ACAT1, acetyl-Coenzyme-A acetyltransferase 1; CT, threshold cycle.

fiber sprouting (30). The results of the present study are in accordance with these findings, however, there are several differences between the two studies. In the present study used a recurrent, rather than prolonged, neonatal seizure model, the experimental rats received 45 induced seizures during nine consecutive days (five seizures/day, minimum 30 min interval). Secondly, RT-qPCR and the $2^{-\Delta C T}$ methods were used in the present study, rather than semi-quantitative PCR. In addition, the present study further evaluated the intervention effects of melatonin on hippocampal mossy fiber sprouting and associated metabolism-associated genes. The results of the present study demonstrated for the first time, to the best of our knowledge that the ZnT-1, CaMKII $\alpha$ and MT-1 genes associated with zinc and calcium transduction, and ACAT1, nSMase and Cathepsin-E, which function as modulators of lipid metabolism, may be of particular importance for the inhibitory effects of melatonin on hippocampal mossy fiber regenerative sprouting. It has been established that a ketogenic diet, in which $>90 \%$ of calories are derived from fat, is an effective treatment for lipid metabolism-associated neurological diseases, including epilepsy (31). Notably, all three of the above-mentioned molecules may be targets for the inhibitory effects of the ketogenic diet on epilepsy. Acetyl-co A acetyltransferase (ACAT1) is a mitochondrial enzyme involved in ketogenic pathway metabolism. ACAT1 performs the final step in ketolysis during the processing of fats. It converts one acetoacetyl-CoA into two molecules of acetyl CoA during ketolysis. Coincidentally, it has been hypothesized that ketone bodies contribute to the anticonvulsant and antiepileptic effects of a ketogenic diet (32). Therefore, the present study hypothesized that the downregulation of ACAT1 mRNA observed in the present study can lead to decreased ketolysis. Therefore, the resulting increase in the level of ketones may trigger the compensatory anticonvulsant effects. In parallel with the dowregulated expression of ACAT1, the present study also observed down- 

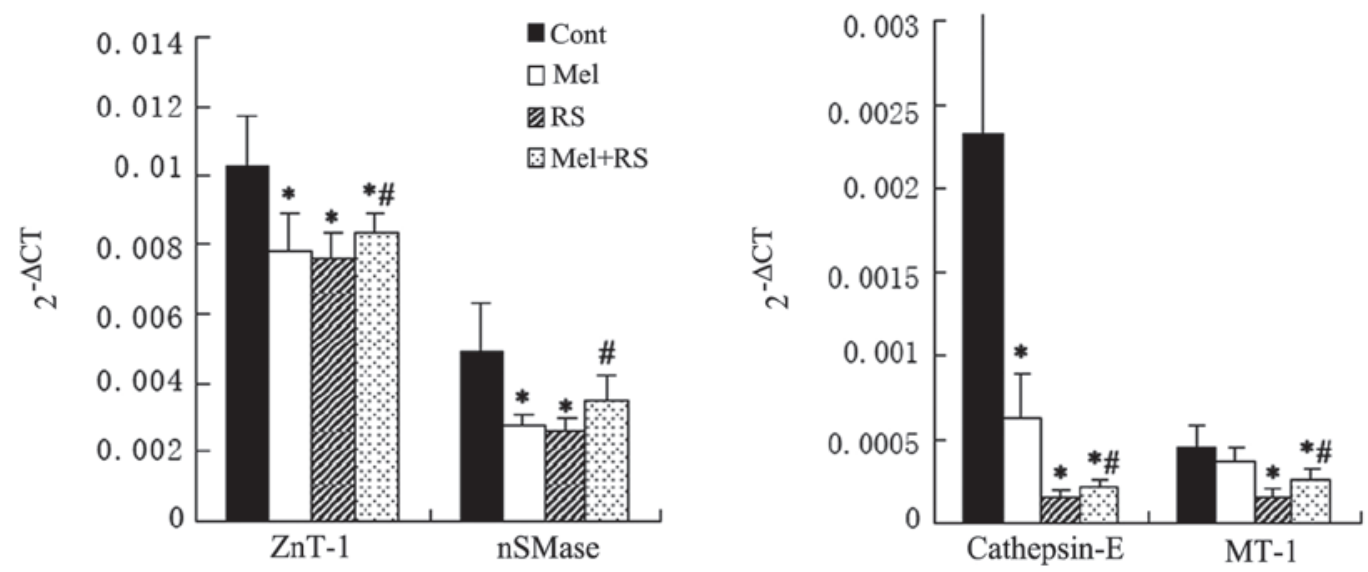

\begin{tabular}{lll}
\hline Gene & $\begin{array}{l}\text { GenBank } \\
\text { accession number }\end{array}$ & Primer sequence \\
\hline ZnT-1 & NM_022853 & $\begin{array}{l}\text { F: 5'- CGTTGTTGTGAATGCCTTGGT-3' } \\
\text { R:5'-GGGTTCACACAAAAGTCGTCTTC-3' } \\
\text { probe::5'-FAM-TTCTACTTTTCCTGGAAGGGTTGTA-TAMRAM-3' }\end{array}$ \\
& & F: 5'- CAGGCTGAGATACAACAT -3' \\
& R: 5'- TCAAGCAAGTTTATTTTCTTC -3' \\
& probe:5'-FAM-CTGACAAGGACAACGGAGACC-TAMRA -3' \\
& & F: 5'- CAGCAATTTGGAGAAAGT -3' \\
& R: 5'- GGCCATCATGTTATCAAA -3' \\
& probe: 5'-FAM-CGACCATCGCACAGATCAGAGACCAG-TAMRA -3' \\
\hline Cathepsin-E & NM_012938 & F: 5'- GACTTGGCACACAACTTCACAGA -3' \\
& & R: 5'- GGACTGTCATCGTGGTCAGGAT -3' \\
& probe: 5'-FAM-CTGGCCATTGGTGCTTCCTTCCG-TAMRA -3'
\end{tabular}

Figure 4. RT-qPCR analysis of hippocampal zinc/lipid-associated genes (ZnT-1, MT-1, nSMase and Cathepsin-E). Notably, the expression levels of the four genes in the RS group were significantly downregulated, compared with those in the Cont group ( $\mathrm{P}<0.05)$, however, the mRNA levels of the four genes were increased in the Mel+RS group, compared with those in the RS group ( $\left.{ }^{\#} \mathrm{P}<0.05\right)$. Data are presented as the mean \pm standard deviation. The lower part of the chart shows the oligonucleotide primers used for RT-qPCR analysis. RT-qPCR, reverse transcription-quantitative polymerase chain reaction; Cont, untreated control; Mel, melatonin treatment; RS, recurrent neonatal seizure; ZnT-1, zinc transporter 1; MT-1, metallothionein 1; nSMase, neutral sphingomyelinase; CT, threshold cycle.

regulated expression levels of Cathepsin-E and nSMase. Cathepsin-E is an aspartic proteinase. Abnormal levels of cathepsin-E have been observed in tumor, senile plaques of Alzheimer's disease and KA-injected rat brains (33-35). Notably, using microarray data and subsequent RT-PCR experiments, Jeong et al demonstrated that the hippocampal expression of cathepsin $\mathrm{E}$ was modulated by a ketogenic diet in a KA-induced seizure model (4). Sphingomyelinase (SMase) is an enzyme responsible for ceramide, ceramide 1-phosphate, sphingosine and sphingosine 1-phosphate production in the brain by catabolizing glycosphingolipids. These metabolites modulate the activity of phospholipase A(PLA) (3), and exogenous PLA (3) can increase ketogenesis, while a ketogenic diet inhibits brain ganglioside GM2 accumulation $(36,37)$. This cross talk between metabolites of glycerophospholipid and sphingolipid metabolism suggests an important role of SMase in the processes of ketogenic diet-mediated neuroprotection following developmental- seizure-induced neuropathology.

Ruiz et al (38) examined the expression levels of energy metabolism-associated genes in the hippocampus of age-matched control and chronic epileptic animals. The results demonstrated that Kcnj11 was significantly upregulated $24 \mathrm{~h}, 1$ month and 2 months post-SE. The results of the present study also revealed downregulated expression levels of Kcnj11, and of Lepr, Drd2 and Mc4r in the hippocampi of rats in the RS group compared with those in the Cont group. Kenj11 is a subunit of the $\mathrm{K}_{\text {АTP }}$ channel, which is involved in neuroexcitability and cognitive function, and mutations in the $\mathrm{K}_{\text {АTP }}$ channel in humans have been linked to developmental delay, epilepsy and neonatal diabetes syndrome (39). Mice lacking this channel are prone to seizures when subjected to brief periods of hypoxia (40). Mc4r, leptin and Drd2 are present in the brain and hypothalamus, which are involved in regulating food intake and energy metabolism. Studies have demonstrated that the three genes can have antiepileptic effects in the brain (41-43). Therefore, the downregulated expression levels of Mc4r, Drd2 and leptin in the RS group in the present study may be associated with seizure propagation during epileptogenesis.

In a previous study by Ueda $e t a l$, genes associated with lipid metabolism, Apoa1, Gh, Mc4r, Oprk1 and Pdk4, in the hippocampus were temporarily upregulated in the sub-chronic phase in a rat model of posttraumatic epilepsy (44). In the present study, abnormal expression patterns were also observed in the Apoa1, Oprk1, Pdk4 and Cyp46a1 lipid metabolism-associated genes, however, these genes were 


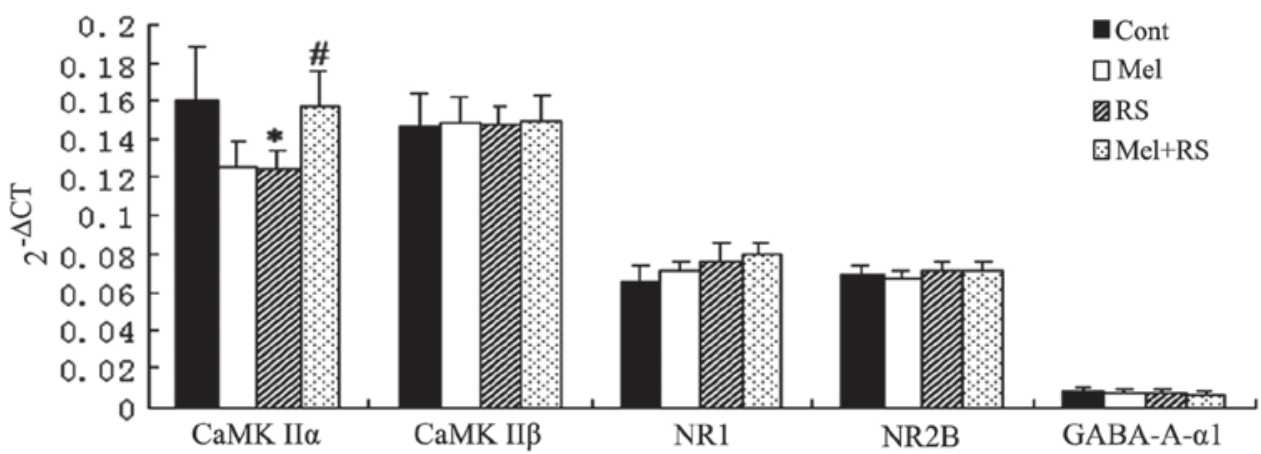

\begin{tabular}{|c|c|c|}
\hline Gene & $\begin{array}{l}\text { GenBank } \\
\text { accession number }\end{array}$ & Primer sequence \\
\hline CaMK II $\alpha$ & NM_012920 & $\begin{array}{l}\text { F:5'-CGAGGGCCTGGACTTTCA-3' } \\
\text { R:5'-TGCACGGGCTTGCTGTT-3' } \\
\text { Probe:5'-FAM-CGATTCTATTTTGAAAACCTGTGGTCCCG-TAMR } \\
\text { A-3' }\end{array}$ \\
\hline CaMK II $\beta$ & NM_001042354 & $\begin{array}{l}\text { F:5'-CATCACCAGCCCCAAAGG-3' } \\
\text { R:5'-TGGCATCTTCGTCCTCTATGG-3' } \\
\text { Probe:5'-FAM-CCTCCTGCCGCCCTGGAATCTT-TAMRA-3' }\end{array}$ \\
\hline NR1 & X63255 & $\begin{array}{l}\text { F: 5'-CGGGTAAACAACAGCAACAAAA-3' } \\
\text { R: 5'-TCCGCTTGGCCACTGAGT-3' } \\
\text { probe::5'-FAM-AGTGGAACGGAATGATGGGCGAGC-TAMRA-3' }\end{array}$ \\
\hline NR2B & NM_012574 & $\begin{array}{l}\text { F:5'-CAACATGCTCTCTCCCTTAATCTG-3' } \\
\text { R: 5'- CAGCTAGTCGGCTCTCTTGGTT-3' } \\
\text { probe: 5'-FAM-CCGCCTAGAGGTTTGGCGTCTA-TAMRA-3' }\end{array}$ \\
\hline $\begin{array}{l}\text { GABA-A } \\
\alpha 1\end{array}$ & AY574250 & $\begin{array}{l}\text { F:5'- TGCGACCATAGAACCGAAAGA- 3, } \\
\text { R:5'-TTGCTGACGCTGTTAAAGGTTTT-3, } \\
\text { Probe:5'-FAM- TCAAGCCTGAGACAAAACCGCCAGA -TAMRA-3' }\end{array}$ \\
\hline
\end{tabular}

Figure 5. RT-qPCR analysis of hippocampal neural excitability-associated genes (CaMKII $\alpha$ and $\beta$, NR1, NR2B and GABA-A- $\alpha 1$ ). Notably, the expression of $\mathrm{CaMKII} \alpha$ in the RS group was significantly downregulated, compared with the Cont group $\left({ }^{*} \mathrm{P}<0.05\right)$, however, the mRNA level was increased in the Mel+RS group, compared with the RS group $\left({ }^{\#} \mathrm{P}<0.05\right)$. Data are presented as the mean \pm standard deviation. The lower part of the chart shows the oligonucleotide primers used for RT-qPCR analysis. RT-qPCR, reverse transcription-quantitative polymerase chain reaction; Cont, untreated control; Mel, melatonin treatment; RS, recurrent neonatal seizure; CaMK II calcium/calmodulin-dependent protein kinase II; NR1, N-methyl-D-aspartate receptor; NR2B, GABA-A- $\alpha 1$, GABA-A receptor $\alpha$; NR2B, N-methyl D-aspartate 2B; CT, threshold cycle.

markedly downregulated in developmental seizures (RS and Mel+RS groups), compared with the Cont group. This discrepancy may be due to the different animal models used or the different time-points analyzed. Ueda et al used adult animals (5 weeks of age) and detected the gene expressions 15 days following amygdale injection (sub-chronic phase of injury), while the present study used a neonatal recurrent seizure model, in which the gene expression levels were detected 30 days following the initial attack (chronic phase of injury).

Notably, while no clear adverse effects were observed in the melatonin-treated animals, certain energy/lipid metabolism-associated genes were markedly downregulated by melatonin, compared with that in the control. Due to a limit number of hippocampal samples, the present study did not further evaluate the changes in the protein expression levels. However, our preliminary investigation revealed that normal rats fed a ketogenic diet had long-term adverse effects on neurobehavioral functions, detected using an open-field assessment (data not shown). Therefore, whether melatonin has long-term adverse effects on brain development and function in normal animals requires further investigation using a variety of toxicology and neurobehavioral methods.

\section{Acknowledgements}

This study was supported by the National Natural Science Foundation of China (nos. 81271458 and 81471337), the Jiangsu Province's Key Provincial Talents Program (no. RC2011113) and a Project Funded by the Priority Academic Program Development of Jiangsu Higher Education Institutions.

\section{References}

1. Tolaymat A, Nayak A, Geyer JD, Geyer SK and Carney PR: Diagnosis and management of childhood epilepsy. Curr Probl Pediatr Adolesc Health Care 45: 3-17, 2015.

2. Anovadiya AP, Sanmukhani JJ and Tripathi CB: Epilepsy: Novel therapeutic targets. J Pharmacol Pharmacother 3: 112-117, 2012.

3. Banach M, Gurdziel E, Jędrych M and Borowicz KK: Melatonin in experimental seizures and epilepsy. Pharmacol Rep 63: 1-11, 2011.

4. Uberos J, Augustin-Morales MC, Molina Carballo A, Florido J, Narbona E and Muñoz-Hoyos A: Normalization of the sleep-wake pattern and melatonin and 6-sulphatoxy-melatonin levels after a therapeutic trial with melatonin in children with severe epilepsy. J Pineal Res 50: 192-196, 2011.

5. Banach M, Gurdziel E, Jedrych M and Borowicz KK: Melatonin in experimental seizures and epilepsy. Pharmacol Rep 63: 1-11, 2011

6. Jain S and Besag FM: Does melatonin affect epileptic seizures? Drug Saf 36: 207-215, 2013. 
7. Ardura J, Andres J, Garmendia JR and Ardura F: Melatonin in epilepsy and febrile seizures. J Child Neurol 25: 888-891, 2010.

8. Paprocka J, Dec R, Jamroz E and Marszał E: Melatonin and childhood refractory epilepsy-a pilot study. Med Sci Monit 16: CR389-396, 2010.

9. Goldberg-Stern H, Oren H, Peled N and Garty BZ: Effect of melatonin on seizure frequency in intractable epilepsy: A pilot study. J Child Neurol 27: 1524-1528, 2012.

10. Sheldon SH: Pro-convulsant effects of oral melatonin in neurologically disabled children. Lancet 351: 1254, 1998.

11. Banach M, Gurdziel E, Jedrych M and Borowicz KK: Melatonin in experimental seizures and epilepsy. Pharmacol Rep 63: 1-11, 2011

12. Musshoff U and Speckmann EJ: Diurnal actions of melatonin on epileptic activity in hippocampal slices of rats. Life Sci 73 : 2603-2610, 2003.

13. Forcelli PA, Soper C, Duckles A, Gale K and Kondratyev A: Melatonin potentiates the anticonvulsant action of phenobarbital in neonatal rats. Epilepsy Res 107: 217-223, 2013.

14. Ni H, Jiang YW, Xiao ZJ, Tao LY, Jin MF and Wu XR: Dynamic pattern of gene expression of ZnT-1, ZnT-3 and PRG-1 in rat brain following flurothyl-induced recurrent neonatal seizures. Toxico Lett 194: 86-93, 2010

15. Ni H, Ren SY and Zhang LL: Expression profiles of hippocampal regenerative sprouting-related genes and their regulation byE-64 $\mathrm{d}$ in a developmental rat model of penicillin-induced recurrent epilepticus. Toxico Lett 217: 162-169, 2013.

16. Ni H, Yan JZ, Zhang LL, Feng X and Wu XR: Long-term effects of recurrent neonatal seizures on neurobehavioral function and related gene expression and its intervention by inhibitor of cathepsin B. Neurochem Res 37: 31-39, 2012.

17. Moezi L, Shafaroodi H, Hojati A and Dehpour AR: The interaction of melatonin and agmatine on pentylenetetrazole-induced seizure threshold in mice. Epilepsy Behav 22: 200-206, 2011.

18. Mareš J, Pometlová M, Deykun K, Krýsl D and Rokyta R: An isolated epileptic seizure elicits learning impairment which could be prevented by melatonin. Epilepsy Behav 23: 199-204, 2012.

19. Ni H, Jiang YW, Tao LY, Cen JN and Wu XR: Effects of penicillin-induced developmental epilepticus on hippocampal regenerative sprouting, related gene expression and cognitive deficits in rats. Toxico Lett 188: 161-166, 2009.

20. Johnson MR, Wang K, Smith JB, Heslin MJ and Diasio RB Quantitation of dihydropyrimidine dehydrogenase expression by real-time reverse transcription polymerase chain reaction. Anal Biochem 278: 175-184, 2000

21. Tian Y, Yabuki Y, Moriguchi S, Fukunaga K, Mao PJ and Hong LJ: Melatonin reverses the decreases in hippocampal protein serine/threonine kinases observed in an animal model of autism. J Pineal Res 56: 1-11, 2014.

22. Koh PO: Melatonin regulates the calcium-buffering proteins, parvalbumin and hippocalcin, in ischemic brain injury. J Pineal Res 53: 358-365, 2012.

23. Cetinkaya M, Alkan T, Ozyener F, Kafa IM, Kurt MA and Koksal N: Possible neuroprotective effects of magnesium sulfate and melatonin as both pre- and post-treatment in a neonatal hypoxic-ischemic rat model. Neonatology 99: 302-310, 2011.

24. Sánchez-Barceló EJ, Mediavilla MD and Reiter RJ: Clinical uses of melatonin in pediatrics. Int J Pediatr 2011: 892624, 2011.

25. Zamorskii II, Sopova IY and Khavinson VKh: Effects of melatonin and epithalamin on the content of protein and lipid peroxidation products in rat cortex and hippocampus under conditions of acute hypoxia. Bull Exp Biol Med 154: 51-53, 2012

26. Tchekalarova J, Petkova Z, Pechlivanova D, Moyanova S, Kortenska L and Mitreva R: Prophylactic treatment with melatonin after status epilepticus: effects on epileptogenesis, neuronal damage and behavioral changes in a kainate model of temporal lobe epilepsy. Epilepsy Behav 27: 174-187, 2013.
27. Jain A, Sharma D, Suhalka P, Sukhwal P and Bhatnagar M: Changes in the density of nitrergic neurons in the hippocampus of rats following kainic acid and melatonin administration. Physiol Res 62: 197-203, 2013.

28. Mareš J, Stopka P, Nohejlová K and Rokyta R: Oxidative stress induced by epileptic seizure and its attenuation by melatonin. Physiol Res 62 (Suppl 1): 67-74, 2013.

29. Atanasova M, Petkova Z, Pechlivanova D, Dragomirova P, Blazhev A and Tchekalarova J: Strain-dependent effects of long-term treatment with melatonin on kainic acid-induced status epilepticus, oxidative stress and the expression of heat shock proteins. Pharmacol Biochem Behav 111: 44-50, 2013.

30. Ni H, Jiang YW, Tao LY, Jin MF and Wu XR: ZnT-1, ZnT-3, CaMK II, PRG-1 expressions in hippocampus following neonatal seizure-induced cognitive deficit in rats. Toxicol Lett 184: 145-150, 2009.

31. Adibhatla RM and Hatcher JF: Altered lipid metabolism in brain injury and disorders. Subcell Biochem 49: 241-268, 2008.

32. McNally MA and Hartman AL: Ketone bodies in epilepsy. J Neurochem 121: 28-35, 2012.

33. Zaidi N, Hermann C, Herrmann T and Kalbacher H: Emerging functional roles of cathepsin E. Biochem Biophys Res Commun 377: 327-330, 2008

34. Bernstein HG and Wiederanders B: An immunohistochemical study of cathepsin E in Alzheimer-type dementia brains. Brain Res 667: 287-290, 1994.

35. Tominaga $\mathrm{K}$, Nakanishi $\mathrm{H}$, Yasuda $\mathrm{Y}$ and Yamamoto $\mathrm{K}$ : Excitotoxin-induced neuronal death is associated with response of a unique intracellular aspartic proteinase, cathepsin $\mathrm{E}$. J Neurochem 71: 2574-2584, 1998.

36. Chihara M, Nomura T, Tachibana M, Nomura H, Nomura Y and Hagino Y: Effects of exogenous phospholipase enzymes, arachidonic acid and 1-oleoyl-2-acetyl-sn-glycerol on ketogenesis in isolated rat hepatocytes. Biochim Biophys Acta 1012: $5-9,1989$

37. Farooqui AA, Horrocks LA and Farooqui T: Interactions between neural membrane glycerophospholipid and sphingolipid mediators: a recipe for neural cell survival or suicide. J Neurosci Res 85: 1834-1850, 2007.

38. Ruiz N, Pacheco LF, Farrell B, Cox CB, Ermolinsky BS, Garrido-Sanabria ER and Nair S: Metabolic gene expression changes in the hippocampus of obese epileptic male rats in the pilocarpine model of temporal lobe epilepsy. Brain Res 1426: 86-95, 2011

39. Koster JC, Cadario F, Peruzzi C, Colombo C, Nichols CG and Barbetti F: The G53D mutation in Kir6.2 (KCNJ11) is associated with neonatal diabetes and motor dysfunction in adulthood that is improved with sulfonylurea therapy. J Clin Endocrinol Metab 93: 1054-1061, 2008

40. Yamada K, Ji JJ, Yuan H, Miki T, Sato S, Horimoto N, Shimizu T, Seino S and Inagaki N: Protective role of ATP-sensitive potassium channels in hypoxia-induced generalized seizure. Science 292: 1543-1546, 2001.

41. Liu ZL, He B, Fang F, Tang CY and Zou LP: Analysis of single nucleotide polymorphisms in the melanocortin- 4 receptor promoter in infantile spasms. Neuropediatrics 38: 304-309, 2007.

42. Dunleavy M, Provenzano G, Henshall DC and Bozzi Y: Kainic acid-induced seizures modulate Akt (SER473) phosphorylation in the hippocampus of dopamine D2 receptor knockout mice. J Mol Neurosci 49: 202-210, 2013

43. Jayaram B, Khan RS, Kastin AJ, Hsuchou H, Wu X and Pan W: Role of astrocytic leptin signaling against excitotoxicity. J Mol Neurosci 49: 523-530, 2013.

44. Ueda Y, Kitamoto A, Willmore LJ and Kojima T: Hippocampal gene expression profiling in a rat model of posttraumatic epilepsy reveals temporal upregulation of lipid metabolism-related genes. Neurochem Res 38: 1399-1406, 2013. 